\title{
On the importance of looking back: The role of recursive remindings in recency judgments and cued recall
}

\author{
Larry L. Jacoby • Christopher N. Wahlheim
}

Published online: 1 February 2013

(C) Psychonomic Society, Inc. 2013

\begin{abstract}
Suppose that you were asked which of two movies you had most recently seen. The results of the experiments reported here suggest that your answer would be more accurate if, when viewing the later movie, you were reminded of the earlier one. In the present experiments, we investigated the role of remindings in recency judgments and cued-recall performance. We did this by presenting a list composed of two instances from each of several different categories and later asking participants to select (Exp. 1) or to recall (Exp. 2) the more recently presented instance. Reminding was manipulated by varying instructions to look back over memory of earlier instances during the presentation of later instances. As compared to a control condition, cued-recall performance revealed facilitation effects when remindings occurred and were later recollected, but interference effects in their absence. The effects of reminding on recency judgments paralleled those on cued recall of more recently presented instances. We interpret these results as showing that reminding produces a recursive representation that embeds memory for an earlier-presented category instance into that of a later-presented one and, thereby, preserves their temporal order. Large individual differences in the probabilities of remindings and of their later recollection were observed. The widespread importance of recursive reminding for theory and for applied purposes is discussed.
\end{abstract}

Keywords Recursive remindings $\cdot$ Recency judgments . Cued recall

Life can only be understood backwards, but it must be lived forwards.

—Søren Kierkegaard (1869/1996)

L. L. Jacoby $(\square) \cdot$ C. N. Wahlheim

Department of Psychology, Washington University in St. Louis,

One Brookings Drive, St. Louis,

Missouri 63130, USA

e-mail:1ljacoby@artsci.wustl.edu
Although life must be lived forward, memory can serve as a source of assistance when dealing with the present and planning for the future. As an example, being reminded of a prior event by the occurrence of a later event can influence the encoding and subsequent memory of both the earlier and the later events (e.g., Wahlheim \& Jacoby, 2013). However, such reminding is not likely to always be spontaneous, but instead may sometimes rely on purposeful looking back to the past. In the present article, we examine the importance of looking back for judgments of recency and for performance when participants are asked to recall the most recently presented instance of a category. For both types of task, we show that looking back, along with resultant remindings, are important for performance and can be brought under task control.

We begin by describing the importance of reminding for a variety of memory tasks, and then consider advantages of bringing remindings under task control as a means of investigating their effects. To anticipate, we show that as compared to a control condition, proactive facilitation is found in cued recall of the most recently presented instance of a category in the presence of recollection of a prior reminding, whereas proactive interference is found in its absence. In the General Discussion, we describe the widespread importance of remindings and discuss results suggestive of large individual differences in the likelihood of looking back, along with the potential importance of those individual differences.

Berntsen, Staugaard, and Sørensen (2013) provided an excellent review of research revealing spontaneous episodic remembering, and they reported results to contrast voluntary with spontaneous remembering. Spontaneous remembering was described as being faster and more dependent on stimulus control than is voluntary memory. Berntsen et al. focused on intrusive recollections such as flashbacks (see also B. A. Anderson, Jacoby, Thomas, \& Balota, 2011, and Ste-Marie \& Jacoby, 1993, for research showing interfering effects of spontaneous remembering). However, spontaneous remembering 
(reminding) also has beneficial effects, as in the case of its contributing to the effects of repetition. An early example of this can be seen in paired-associate learning experiments by Asch, Rescorla, and Linder, as reported by Asch (1969). In their experiments, a single well-learned pair from a first list was included in a second list of pairs that was presented after a delay. This form of presentation discouraged participants' recognition of the repetition in List 2, resulting in only a small percentage of participants doing so. Participants who did not recognize the repeated pair as being such showed no advantage in memory for the repeated pair, as compared to the "new" pairs that only appeared in List 2. In contrast, participants who did recognize the repetition showed a facilitative effect. Furthermore, when another group was told about the repetition and encouraged to notice it prior to studying List 2 , nearly every participant did so, and also showed a facilitative effect of repetition. Results similar to those reported by Asch et al. have been reported by others and described as evidence of the importance of detecting repetitions along with retrieval processes for finding a facilitative effect of spacing repetitions (e.g., Appleton-Knapp, Bjork, \& Wickens, 2005; Bellezza, Winkler, \& Andrasik, 1975, Exp. 3; Benjamin \& Tullis, 2010; Braun \& Rubin, 1998, Exp. 2).

In addition to being important for repetition effects, remindings that rely on the detection of relationships among events are important for preserving the temporal order of events. Judgments of recency are superior for related (e.g., queen-king) as compared to unrelated (e.g., spider-table) words (Hintzman, 2010; Tzeng \& Cotton, 1980; Winograd \& Soloway, 1985). This effect on memory for temporal order has been explained as being produced by the presentation of the second member of a related pair reminding participants of the first member of the pair (Hintzman, 2011; Tulving, 1983, p. 42; Winograd \& Soloway, 1985). It has been argued that reminding results in a recursive representation in which the first-presented member of a related pair is embedded in the memory of the second-presented member. A recursive representation creates a memory dependence between the members of a related pair and preserves information about their temporal order. For example, if asked which occurred more recently, queen or king, recursive reminding allows the question to be answered by recollecting that the study presentation of queen reminded one of the earlier-presented king, and so, queen must have occurred more recently. Importantly for the present purposes, Hintzman (2011) described recursive remindings as generally being automatic (spontaneous). Although we agree that recursive remindings are sometimes spontaneous, we will show here that recursive reminding can be brought under task control. Later, we will consider the advantages of doing so.

Wahlheim and Jacoby (2013) showed the importance of recursive reminding for proactive effects of memory on cued recall in an A-B, A-D paradigm. Participants in their experiments studied two lists of word pairs that included pairs with the same cue and a changed response (A-B, A-
D), and control pairs presented exclusively in the second list (A-B, C-D). At test, participants were provided with the left-hand member of the pairs as a cue for recall of the righthand member presented in the second list. The results revealed that performance on $\mathrm{A}-\mathrm{D}$ pairs reflected a mixture of facilitation and interference effects as compared to the control condition. Proactive facilitation occurred when the change in response in the second list was detected and later recollected, whereas proactive interference occurred when change was not detected or was detected but not recollected at test.

The results above show that proactive effects of memory depended on the detection and recollection of change and can be interpreted as showing the importance of recursive reminding. The finding of opposite effects being dependent on the presence or absence of recollection provides support for a distinction between recollection and automatic influences of memory (see Yonelinas \& Jacoby, 2012, for a review of evidence supporting that distinction). We will further describe procedures employed by Wahlheim and Jacoby (2013) when introducing Experiment 2, which extended those procedures to recall cued with category names. Also, as will be described, the results reported by Wahlheim and Jacoby revealed large individual differences in the detection of change that were important for later memory performance. We sought similar evidence in Experiment 2.

The experiments reported here explored the extent to which looking back and resultant recursive remindings can be brought under task control. To do so, we employed a variant of a looking-back procedure that had been used by Jacoby (1974). ${ }^{1}$ In his experiments, pairs of category instances (e.g., dog, horse) were presented, with members of a pair being separated by various spacings (e.g., zero, one, three, and seven). Also included in the study list were single items for which only the second member of the pair (e.g., horse) was presented. In an $n$-back condition, participants were instructed to respond "yes" if the current instance was from the same category as any instance presented earlier in the experiment, regardless of the distance back of the earlier instance. These instructions were meant to encourage looking back across the whole list and thereby to promote study-phase retrieval (reminding) of the first member of a pair during presentation of the second member, regardless of the spacing of the instances. In contrast, participants in a one-back condition were instructed to respond "yes" only if the current instance was from the same category as the item that had immediately preceded it in the list. For this group, the instructions were meant to restrict remindings to immediately preceding items,

\footnotetext{
${ }^{1}$ The Jacoby (1974) article was originally titled "The Importance of Looking Back." However, the editor of the journal in which it was published objected, saying that the title was too mentalistic and demanded that it be changed
} 
with the consequence that remindings would not occur for instances presented at longer spacings.

At test, participants were presented with the firstpresented instance of each studied category (e.g., $d o g$ ) as a cue for recall of the second instance or, in the case of single items, for recall of the only instance of the category that had been presented. That is, the recall cue was an extralist cue for single items but an intralist cue for items included in pairs. The results revealed strong evidence of the importance of looking back by showing very different spacing functions for the $n$-back and one-back conditions. For the $n$ back condition, cued recall remained high across all spacings. In contrast, for the one-back condition, cued-recall performance was high at spacing 0 but then dropped to near the level of single items at longer spacings. These results provided evidence that remindings seldom, if ever, occurred spontaneously for exemplars presented with longer spacings in the one-back condition, and so, an intralist cue was little more effective than was an extralist cue. A third condition, in which participants were simply instructed to learn the presented list of words and were not required to explicitly judge the relationship between items, produced results that were intermediate between those of the one- and $n$-back conditions. That result was interpreted as evidence of individual differences in the probability of looking back. It was suggested that when simply instructed to learn, looking back by some participants was similar to that of those in the oneback condition, whereas others looked farther back, as in the $n$-back condition.

We designed Experiment 1 to show that recursive reminding that is important for recency judgments can be brought under task control by means of looking-back instructions. Pairs of words from categories were separated by 0,3 , or 12 intervening words, with the one-back and $n$ back conditions being the same as those employed by Jacoby (1974). At test, both members of pairs were presented, and participants were instructed to select the word from each pair that had been presented more recently. At spacing 0 , we expected to find no difference in the accuracy of recency judgments for the one-back and $n$-back conditions, because recursive remindings would occur in both conditions. However, for longer spacings, we expected recursive reminding and the resultant accuracy of recency judgments to remain high for the $n$-back condition and to drop for the one-back condition, showing a pattern of results the same as had been reported by Jacoby. Such results would provide evidence that recursive reminding can be brought under task control by showing that the presence of a relationship between items from a pair is not sufficient to enhance later recency judgments, but rather, the task must be such that it encourages recursive reminding.

In Experiment 2, we employed a procedure similar to that used in Experiment 1, but rather than requiring recency judgments, we provided participants with the category names as cues and asked them to recall the most recently presented member of each category. Doing so allowed us to examine the relation between recency judgments and category-cued recall of the most recently presented items. As will be shown, the results extended those reported by Wahlheim and Jacoby (2013) by revealing a mix of proactive facilitation and proactive interference. When recursive reminding was recollected, proactive facilitation was observed, whereas proactive interference was observed when recollection of reminding was absent. The results also revealed the importance of individual differences in remindings for later memory performance.

Hintzman (2011) suggested that remindings are typically spontaneous. However, bringing remindings under task control has advantages for investigating their effects and for exploring the utility of the distinction between spontaneous and cognitively controlled remindings. The procedures employed in investigations of the effects of reminding on recency judgments (e.g., Hintzman, 2010; Tzeng \& Cotton, 1980; Winograd \& Soloway, 1985) have not provided evidence that remindings were spontaneous. In those experiments, the manipulation of reminding was confounded with materials (related vs. unrelated items), and reminding occurred under instructions to remember the presented items. Perhaps instructions to study items and remember them for a later test are sufficient to encourage the detection of relationships among presented items. That is, remindings may not have been spontaneous, in the sense of being independent of the task in which participants were engaged. In contrast, our looking-back procedure held the materials constant across conditions and allowed us to examine performance in the one-back condition to determine whether the presence of a category relationship between items was sufficient to produce spontaneous remindings that were discouraged by the task instructions. Evidence of such spontaneous reminding would be revealed by participants in the one-back condition finding it difficult to reject the second-presented member of pairs as being related to the earlier-presented one when the spacing of the pair members was nonzero. Furthermore, for recency judgments, spontaneous reminding would result in the pattern of results for the one-back condition being similar to that for the $n$-back condition.

Bringing reminding under task control is particularly advantageous for investigating the effects of remindings on cued-recall performance. Our earlier experiments that showed the importance of recollection of remindings for proactive effects of memory (Wahlheim \& Jacoby, 2013) relied upon results gained by conditionalizing the probability of cued recall on a measure of recollection of reminding, giving reason for concern that item differences due to conditionalizing contributed to the results. Bringing remindings under task control makes it unnecessary to use conditionalized data to examine 
their effects. Also, our looking-back procedure makes it possible to reveal parallels between the effects of reminding on recency judgments and cued-recall performance. As was described above, we expected such parallels to be observed and to provide evidence that recursive reminding preserved temporal-order information that was important for both tasks. In the General Discussion, we will contrast the recursivereminding account of proactive effects with traditional accounts that appeal to differences in list discrimination (e.g., Postman \& Underwood, 1973).

\section{Experiment 1}

\section{Method}

Participants A group of 56 Washington University students participated in exchange for course credit or $\$ 10 / \mathrm{h}$. The participants were randomly assigned to either the one-back or the $n$-back condition, resulting in 28 participants per group. The data from three participants in the $n$-back condition were removed and replaced due to obvious failure to follow instructions during the study phase.

Design and materials A 2 (looking back: one-back vs. $n$ back) $\times 4$ (spacing: 0 vs. 3 vs. 12 vs. single items) mixed factorial design was used. Looking back was manipulated between subjects, and spacing was manipulated within subjects.

The materials consisted of two instances from each of 57 categories (e.g., category $=$ a metal; instances $=$ steel and iron). Forty categories were used for critical items, 12 were used for fillers that were not later tested, and five were used for buffers against primacy and recency effects. Instances were chosen from the top five most frequently reported instances in their respective categories, according to the norms reported by Van Overschelde, Rawson, and Dunlosky (2004). The 40 critical categories were divided into four groups of ten categories each, which appeared equally often in each spacing condition and in the single-item condition across participants. Filler and buffer items remained constant across formats. The study list contained 104 items. One instance from each buffer category pair appeared at the beginning (five items) and end (five items) of the list (ten items total). Seventy critical items (20 from each spacing condition, which included two instances from each of ten categories, and ten from the single-item condition, which included one instance from each of ten categories) and 24 filler items (eight from each spacing condition, including two instances per category, and no single items) were intermixed throughout. At test, the ten buffer instances formed five practice pairs, and the 70 critical instances, plus ten previously unstudied instances from single-item categories, formed 40 test pairs. The assignments of categories to spacing conditions and the single-item condition were counterbalanced such that each critical category appeared in each condition equally often across participants. For the single-item condition, the second-presented instances from categories were rotated through conditions across formats, whereas the first-presented instances were not.

Procedure In the study list, critical and filler items appeared individually in a fixed random order that was constrained such that instances from the same spacing condition did not appear consecutively more than three times. In the one-back condition, participants were instructed to respond "yes" when an instance was from the same category as the immediately preceding item, and to respond "no" when the preceding instance was from a different category. In the $n$-back condition, participants were instructed to respond "yes" when an instance was from the same category as any preceding item in the list. Participants responded by clicking on boxes labeled "yes" and "no" displayed below the instances. The study items appeared on the screen for $5 \mathrm{~s}$, regardless of whether a response was made. Participants were told to use the time remaining after making their judgments to study the instances for an upcoming test.

At test, pairs of instances belonging to the same category were presented in a fixed random order. The more recently presented member of a pair appeared equally often on the right and the left. Participants were told to click on the instance that had appeared more recently and were informed that for some pairs (single items) only one instance had been presented earlier, and that they should click on those instances. For all items, participants were not allowed to respond until $3 \mathrm{~s}$ had passed, at which time the border surrounding each instance changed from white to yellow. This delay was meant to discourage the use of familiarity as a basis for responding.

\section{Results and discussion}

The significance level for all tests was set at $p<.05$. Main effects are not reported when they are qualified by significant interactions.

Looking back The top rows of Table 1 show the probabilities of "yes" judgments made for the one-back and $n$-back conditions during study. Analysis of the results from the pairs that appeared during study revealed a significant Spacing $\times$ Looking Back interaction, $F(2,108)=327.39$, $\eta_{\mathrm{p}}{ }^{2}=.86$. The probability of "yes" responses was higher in the $n$-back than in the one-back condition at each level of spacing, but that difference was much larger at spacings 3 and 12 than at spacing 0 , showing that participants followed instructions and were generally accurate in detecting shared 
Table 1 Experiments 1 and 2: Probabilities of "yes" judgments at study as a function of spacing and looking back

\begin{tabular}{lllll}
\hline Looking Back & \multicolumn{2}{l}{ Spacing } & Single Items \\
\cline { 2 - 3 } & 0 & 3 & 12 & \\
\hline Experiment 1 & & & & \\
One-back & $.87(.02)$ & $.09(.02)$ & $.04(.01)$ & $.03(.01)$ \\
$n$-back & $.94(.01)$ & $.88(.02)$ & $.84(.03)$ & $.30(.04)$ \\
Experiment 2 & & & & \\
One-back & $.90(.03)$ & $.06(.05)$ & $.01(.03)$ & $.02(.01)$ \\
$n$-back & $.90(.03)$ & $.80(.05)$ & $.80(.03)$ & $.27(.04)$ \\
\hline
\end{tabular}

Standard errors of the means are presented in parentheses.

category membership. Analysis of the single items showed that "yes" responses were made more often in the $n$-back than in the one-back condition, $t(54)=6.84$. This difference, along with the difference at spacing 0 , suggests that the $n$ back instructions produced a stronger bias to respond "yes." A difference in response bias was expected, because the likelihood of an item requiring a "yes" response was nearly three times higher in the $n$-back than in the one-back condition. An analysis of the probability of a "no" response to first-presented instances provided further evidence of a difference in response bias, by revealing a significant effect of looking-back condition, $F(1,54)=47.06, \eta_{\mathrm{p}}^{2}=.47$. For both of the looking-back conditions, participants should have responded "no" to first-presented instances, but the probability of a "no" response was much higher in the one-back than in the $n$-back condition, providing evidence of a response bias toward responding "yes" in the $n$-back condition. Neither the main effect of item type nor the interaction of looking-back condition with item type was significant in the analysis of "no" responses to firstpresented instances. This lack of effects shows that, as would be expected, responding to single items was generally comparable to responding to the first-presented members of pairs, regardless of the spacing of second-presented members.

Response times for the judgments made during the study phase provided an additional manipulation check for the effectiveness of looking-back instructions. For both looking-back conditions, participants should respond "no" to the first-presented exemplar of a category and to single items, but doing so was expected to be faster in the one-back than in the $n$-back condition. For the one-back condition, the category membership of a presented item only needed to be compared to that of the immediately preceding item, whereas the $n$-back condition required the detection of shared category membership with distantly presented items. Response times for correctly responding "no" were trimmed by deleting times that were 2.5 standard deviations above or below each participant's mean response time, so as to eliminate effects of outliers. As expected, analyses revealed that response times were longer in the $n$-back $(2,182 \mathrm{~ms})$ than in the one-back $(1,323 \mathrm{~ms})$ condition, $F(1,54)=35.57, \eta_{\mathrm{p}}{ }^{2}=$ .40. Furthermore, the interaction of looking-back condition and item type was significant, $F(3,162)=2.99, \eta_{\mathrm{p}}{ }^{2}=.05$. Analyses of that interaction revealed that response times did not differ among item types in the $n$-back condition, $F(3$, $81)=1.72, p=.17, \eta_{\mathrm{p}}{ }^{2}=.06$, but were faster for single items than for all other item types in the one-back condition, $t \mathrm{~s}(27)$ $\geq 2.31, p \mathrm{~s} \leq .03$. We have no interpretation for this interaction, and it was not replicated in Experiment 2.

Differences between the looking-back conditions in response times to the second-presented instance of a category are difficult to interpret, because at nonzero spacings of category exemplars, participants in the one-back condition should respond "no," whereas those in the $n$-back condition should respond "yes" regarding the shared category membership of a presented item. Analysis of response times to the second-presented exemplar of a category revealed only a significant effect of spacing, $F(2,108)=3.45, \eta_{\mathrm{p}}^{2}=.06$. Response times to the second-presented exemplar of a category were faster at spacing $0(1,338 \mathrm{~ms})$ than at spacing 3 $(1,482 \mathrm{~ms}), t(55)=-2.36, p=.02$, but not than at spacing 12 $(1,427 \mathrm{~ms}), t(55)=-1.58, p=.12$.

Recency judgments Table 2 shows that performance on single items (recognition memory) did not differ between looking-back conditions. More importantly, we found a significant Spacing $\times$ Looking Back interaction for pairs, $F(2,108)=4.05, \eta_{\mathrm{p}}{ }^{2}=.07$, that revealed a tendency for performance to increase across spacings in the $n$-back condition and to decrease across spacings in the one-back condition. Follow-up $t$ tests showed that in the one-back condition, performance at spacing 0 was higher than performance at spacings 3 and $12, t \mathrm{~s}(27)>2.11$. At spacing 12 , the accuracy of recency judgments was much higher in the $n$ back condition than in the one-back condition, $t(54)=4.30$,

Table 2 Correct response probabilities for recency judgments (Exp. 1) and cued recall (Exp. 2) as a function of spacing and looking back

\begin{tabular}{lllll}
\hline Looking Back & \multicolumn{2}{l}{ Spacing } & \multicolumn{2}{c}{ Single Items } \\
\cline { 2 - 4 } & 0 & 3 & 12 & \\
\hline Recency Judgments & & & \\
One-back & $.71(.03)$ & $.61(.03)$ & $.61(.03)$ & $.93(.02)$ \\
$n$-back & $.76(.03)$ & $.79(.04)$ & $.80(.03)$ & $.95(.02)$ \\
Cued Recall & & & & \\
One-back & $.53(.05)$ & $.45(.04)$ & $.37(.04)$ & $.60(.04)$ \\
$n$-back & $.54(.05)$ & $.55(.04)$ & $.58(.04)$ & $.73(.04)$ \\
\hline
\end{tabular}

Standard errors of the means are presented in parentheses. 
providing evidence that recursive remindings that resulted from looking back preserved information about temporal order.

The looking-back procedure was successful as a means of bringing reminding under task control. Participants in the one-back condition seldom mistakenly responded "yes" when the second-presented member of a pair occurred at nonzero spacing, and the pattern of results for recency judgments was much different for the one-back than for the $n$ back condition. These results show that the relationship among items alone was not sufficient to produce reminding that was spontaneous, in the sense of being independent of the task in which participants were engaged.

Recollecting that one has been reminded of an earlierpresented item from the same category during the presentation of an item provides a sure basis for recency judgments. For example, if one remembers being reminded of $d o g$ by the presentation of horse, he or she can be certain that dog was presented prior to horse. As an alternative to a recursive-reminding account of our results, it might be argued that in the $n$-back condition, presentation of a second instance of a category often resulted in retrieval of the firstpresented instance of the category, and that its doing so served to "lock in" the list position of the first-presented instance. Against an account of that sort, it could as well be argued that retrieval of the first-presented instance during the presentation of the second-presented instance of a category would reduce their temporal separation, and so result in the instances being associated with similar list contexts, causing increased confusion with regard to their temporal order. Furthermore, retrieval of the first-presented instance might be expected to increase its familiarity, and so increase the difficulty of distinguishing between the recency of the first- and second-presented exemplars on the basis of their familiarity. In contrast, according to the recursive-reminding account, the recursive representation that results from reminding, rather than the effects of retrieval per se, is what is responsible for effects on later recency judgments. Again, the recursive representation preserves both the subject and the object, along with their temporal order in a reminding.

\section{Experiment 2}

The results from Experiment 1 provided evidence of the importance of looking back for recursive remindings that preserve information about temporal order. Experiment 2 extended those findings to predict proactive effects of memory in cued-recall performance. The looking-back conditions and other details of the procedure were the same as in Experiment 1. However, at test, participants were presented with the names of categories from which instances had been presented, and they were instructed to recall the most recently presented member of that category. The form of this test is similar to that of tests used to examine proactive effects of memory - for example, by asking participants to recall responses from a second list after having learned a first list (for a review, see M. C. Anderson \& Neely, 1996). Proactive interference would be evidenced by the accuracy of recall of the second-presented instance of category pairs (P2) being lower than that of single items because of an increase in intrusion errors produced by mistaken recall of the first-presented instance of a category (P1). In line with the results of Experiment 1, we expected recursive remindings to preserve information about temporal order, and thereby protect participants from response competition that would produce such intrusion errors. Consequently, the effects of looking-back instructions on cued-recall performance in Experiment 2 were predicted to be the same as those found for recency judgments in Experiment 1.

To gain further evidence of the importance of looking back for recursive remindings, we employed a variant of the remindings-report procedure used by Wahlheim and Jacoby (2013). Participants were instructed that if another word came to mind prior to or simultaneously with a word that they produced as being the more recently presented category instance, they were to report the word that came to mind. Reporting P1 as having come to mind prior to the report of P2 was treated as indicating that a reminding had occurred when P2 was presented for study and that the reminding was recollected at test. The rationale underlying this procedure is that if study list reminding is recollected at test, the object of the reminding (P1) is expected to come to mind prior to or simultaneously with the event that gave rise to the reminding (P2).

Because of the dependency created by $\mathrm{P} 1$ being embedded in memory for $\mathrm{P} 2$ in a recursive representation, we expected P1 to come to mind along with P2 more often in the $n$-back than in the one-back condition when the study spacing was nonzero. Also, in line with results reported by Wahlheim and Jacoby (2013), we expected to find a mix of proactive facilitation and proactive interference. Proactive facilitation of $\mathrm{P} 2$ recall as compared to the recall of single items was expected to be found when remindings were recollected, but proactive interference was expected when remindings were not recollected at test. Again, these predictions were made because recursive remindings are held to create a dependence between the recall of $\mathrm{P} 1$ and $\mathrm{P} 2$, as well as preserving information about temporal order. In the absence of recursive remindings, $\mathrm{P} 1$ and $\mathrm{P} 2$ are held to compete as potential responses, producing proactive interference.

Method

Participants A group of 48 Washington University students participated in exchange for course credit or $\$ 10 / \mathrm{h}$. The 
participants were randomly assigned to either the one-back or the $n$-back condition, resulting in 24 participants per group.

Design, materials, and procedure The design, materials, and procedure in Experiment 2 were identical to those of Experiment 1, with the following exceptions. At the time of test, unstudied category labels (e.g., a metal) corresponding to studied instances appeared as cues for recall. Participants were instructed to recall either the more recently presented instance from a pair belonging to the named category or the only instance presented, for single items. They were told to make a response for every item, guessing if necessary. Next, they were instructed to report whether another word came to mind prior to or simultaneously with the response that they reported as being most recently presented, and to type that word in a field on the screen. There were no time limits for the cued-recall or remindings-report responses.

\section{Results}

Looking back The bottom rows of Table 1 show that the results replicated those of Experiment 1. The probabilities of a "yes" response to the pairs did not differ in the one-back and $n$-back conditions at spacing 0 , but they were much higher in the $n$-back condition at the longer spacings, $F(2$, $92)=160.35, \eta_{\mathrm{p}}{ }^{2}=.78$. Also replicating Experiment 1 , the probability of responding "yes" to single items was higher in the $n$-back condition than in the one-back condition, $t(46)$ $=6.49$. Finally, responding was comparable for single items and for the first-presented instance of pairs. An analysis of the probability of a "no" response to first-presented instances revealed a significant effect of looking-back condition, $F$ $(1,54)=30.57, \eta_{\mathrm{p}}{ }^{2}=.40$. The probability of a "no" response to first-presented instances and single items was lower in the one-back than in the $n$-back condition. As we noted for Experiment 1, these results provide evidence of a greater bias toward responding "yes" in the $n$-back than in the one-back condition. In contrast to the results of Experiment 1, the analysis of "no" responses revealed a significant interaction between item type and looking-back condition, $F(3,138)=3.79, \eta_{\mathrm{p}}{ }^{2}=.08$. That interaction resulted from the difference between looking-back conditions being somewhat larger for single items than for the pair conditions, and is not easily interpretable. Nevertheless, as would be expected, responding to single items was generally comparable to responding to the first-presented members of pairs, just as we had found in Experiment 1.

As in Experiment 1, response times for "no" responses to first-presented category exemplars and single items were longer in the $n$-back $(2,173 \mathrm{~ms})$ than in the one-back $(1,266 \mathrm{~ms})$ condition, $F(1,46)=54.03, \eta_{\mathrm{p}}{ }^{2}=.54$.
Analysis of response times to the second-presented exemplar of a category revealed a significant interaction between looking-back conditions and spacing, $F(2,90)=3.89, \eta_{\mathrm{p}}{ }^{2}=$ .08 . Note that the data from one participant in the $n$-back condition were not included in analyses of second-presented instances because of failure to respond "yes" at least once in two of the cells. Analyses of that interaction revealed that response times increased with increases in spacing for the $n$ back condition $(1,323,1,484$, and $1,468 \mathrm{~ms}), F(2,44)=$ $4.65, \eta_{\mathrm{p}}{ }^{2}=.18$. In the one-back condition, response times to the second-presented instance of a category did not vary significantly across increases in spacing, $F<1$. This interaction can be understood as resulting from the difficulty of the retrieval involved in reminding increasing with spacing in the $n$-back condition.

Cued recall The results presented in Table 2 show that cued-recall performance for single items was higher in the $n$-back than in the one-back condition, $t(46)=2.28$. Perhaps this was because the potential category memberships of items were more thoroughly processed when preparing for the $n$-back task, making it more likely that the experimenternamed category would be encoded, and so would serve as an effective cue. The difference between looking-back conditions in the response times to first-presented instances and to single items during the study phase is consistent with this possibility, as well as with a difference in response bias.

Analyses of the probability of correctly recalling the second-presented category instances (P2) produced a significant Spacing $\times$ Looking Back interaction for pairs, $F(2,92)$ $=5.13, \eta_{\mathrm{p}}{ }^{2}=.10$. The form of the interaction was the same that we had found in Experiment 1 for recency judgments. Performance was nearly identical for the two looking-back conditions at spacing 0 . We observed a tendency for performance to increase across spacings in the $n$-back condition and to decrease across spacings in the one-back condition. Follow-up $t$ tests showed that in the one-back condition, performance at spacing 0 was higher than performance at spacing $3, t(23)=1.72$ (one-tailed), and at spacing $12, t(23)$ $=3.99$. At spacing 12 , the probability of correctly producing P2 as a response was much higher in the $n$-back than in the one-back condition, $t(46)=3.52$, just as had been found for recency judgments in Experiment 1. These results provide evidence that recursive remindings enhanced cued recall of P2 and were brought under task control by means of the looking-back procedure.

Analysis of the probability of producing P1 as an intrusion when participants were instructed to recall P2 (Table 3) revealed a significant Spacing $\times$ Looking Back interaction for pairs, $F(2,92)=6.13, \eta_{\mathrm{p}}{ }^{2}=.12$. The probability of producing $\mathrm{P} 1$ as an intrusion error decreased across spacings in the $n$-back condition and increased across spacings in the one-back condition. At spacing 12, the spacing at which the 
Table 3 Experiment 2: Probabilities of intrusions of first-presented instances (P1) in cued recall as a function of spacing and looking back

\begin{tabular}{lllll}
\hline Looking Back & \multicolumn{2}{l}{ Spacing } & \multirow{2}{*}{ Single Items } \\
\cline { 2 - 4 } & 0 & 3 & 12 & \\
\hline One-back & $.31(.04)$ & $.28(.04)$ & $.43(.04)$ & $.03(.01)$ \\
$n$-back & $.33(.04)$ & $.30(.04)$ & $.26(.04)$ & $.04(.01)$ \\
\hline
\end{tabular}

The intrusion probabilities for single items reflect baseline rates of responding with first-presented instances from pairs that had not been presented during study. Standard errors of the means are presented in parentheses.

difference between the looking-back conditions was greatest for cued recall in the present experiment and for recency judgments in Experiment 1, the probability of P1 being produced as an intrusion error was much lower in the $n$ back than in the one-back condition, $t(46)=-2.88$.

The probability of recalling the most recently presented instance of a category was lower than that of recalling a single item from a category, providing evidence that response competition between $\mathrm{P} 1$ and $\mathrm{P} 2$ reduced the probability of correct responding (cf. M. C. Anderson \& Neely, 1996). Recursive reminding in the $n$-back condition served to diminish but not eliminate such proactive interference at longer spacings. As will be shown, the failure to eliminate proactive interference occurred because recursive remindings were not always recollected at the time of test. The correspondence between the results of Experiments 1 and 2 provides compelling evidence that recursive remindings enhanced cued-recall performance by preserving information about the order in which category instances had been presented, just as had been found for recency judgments.

Further evidence of the importance of recursive remindings for cued-recall performance was provided by the results from the remindings-report procedure. Table 4 shows that for pairs, $\mathrm{P} 1$ was more likely in the $n$-back than in the oneback condition to be reported as having come to mind prior to the output of a response that was given as being P2, with the difference between conditions increasing with spacing, $F(2,92)=6.91, \eta_{\mathrm{p}}{ }^{2}=.13$. The converse case, of $\mathrm{P} 2$ being reported as coming to mind prior to the erroneous output of

Table 4 Experiment 2: Probabilities of first-presented instances (P1) being reported as coming to mind prior to or simultaneously with recalled responses, as a function of spacing and looking back

\begin{tabular}{lllll}
\hline Looking Back & \multicolumn{2}{l}{ Spacing } & \multirow{2}{*}{ Single Items } \\
\cline { 2 - 4 } & 0 & 3 & 12 & \\
\hline One-back & $.25(.04)$ & $.13(.03)$ & $.10(.04)$ & $.02(.01)$ \\
$n$-back & $.26(.04)$ & $.22(.03)$ & $.29(.04)$ & $.01(.01)$ \\
\hline
\end{tabular}

Standard errors of the means are presented in parentheses. a different response as being the more recently presented, rarely occurred, and its probability of occurrence did not differ between the looking-back conditions (.06 vs. .04). The finding that P1 came to mind prior to P2 more often in the $n$-back condition provides evidence of the validity of the remindings-report procedure as a means of measuring recollection of remindings.

To explore the reliance of proactive effects of memory on recollection of remindings, we compared two probabilities: the probability of correct recall conditionalized on $\mathrm{P} 1$ being reported as coming to mind prior to the response that was produced as being $\mathrm{P} 2$, and the probability of correct recall conditionalized on no other response being reported as having come to mind (Table 5). When P1 was reported as having come to mind, the probability of correct recall was higher than the probability of correct recall for single items (.85 vs. .66), showing proactive facilitation, $t(45)=4.53$, and the accuracies of responding did not differ for the oneback and $n$-back conditions (.87 vs. .83 ), $t<1$. However, the probability of correct responding was lower in the one-back than in the $n$-back condition when no other response was reported as coming to mind, $F(1,46)=6.09, \eta_{\mathrm{p}}{ }^{2}=.12$, and was also lower for items coming from pairs than for single items, $F(1,46)=52.82, \eta_{\mathrm{p}}{ }^{2}=.53$, showing proactive interference. The interaction was not significant, $F<1$. As is suggested for the effect of looking-back condition on the recall of single items, differences in the processing of the potential category memberships of studied items might explain the poorer cued recall of items in the one-back as compared to the $n$-back condition when remindings were not recollected.

The results above agree with those reported by Wahlheim and Jacoby (2013), in showing that the recollection of remindings, as measured by the remindings-report procedure, produces proactive facilitation, whereas failure to recollect remindings produces proactive interference. However, these conclusions are based on results that rely on analyses of conditional probabilities that measure the recollection of remindings by means of the remindings-report procedure. Reliance on analyses of conditional probabilities raises the

Table 5 Experiment 2: Correct-response probabilities for cued recall, conditionalized on report of responses coming to mind prior to or simultaneously with recalled responses, as a function of looking back

\begin{tabular}{llll}
\hline Looking Back & \multicolumn{2}{l}{ Spacing (All Pairs) } & \multirow{2}{*}{ Single } \\
\cline { 2 - 3 } & P1 Reported & No Other Reported & \\
\hline One-back $(N=24)$ & $.87(.04)$ & $.39(.03)$ & $.60(.04)$ \\
$n$-back $(N=22)$ & $.83(.05)$ & $.51(.04)$ & $.73(.04)$ \\
All $(N=46)$ & $.85(.03)$ & $.44(.03)$ & $.66(.03)$ \\
\hline
\end{tabular}

$\mathrm{P} 1=$ the first-presented instance of a category pair. Standard errors of the means are presented in parentheses. 
possibility that item-selection effects rather than effects of recursive reminding were responsible for the results. That is, it might be argued that use of the remindings-report procedure simply served to select the items that were remembered best. The full pattern of our results provides strong evidence against that argument. The results from the reminding-report procedure converge with those from the looking-back conditions in showing the importance of recursive reminding for subsequent cued-recall performance. Conditionalizing results was not required for reaching our conclusions based on varying looking back by means of instructions. Furthermore, the probability of $\mathrm{P} 1$ being reported as having come to mind prior to the response that was output - our remindings-report measure - was higher in the $n$-back than in the one-back condition, as was the probability of correct recall of $\mathrm{P} 2$. This correspondence of results would be expected from a recursive-reminding account but would be difficult to explain in terms of item-selection effects. Nonetheless, we followed Wahlheim and Jacoby (2013) by using a hierarchical regression analysis to gain further evidence that the effects attributed to recursive reminding were not, instead, produced by item-selection effects.

A hierarchical regression analysis was conducted by collapsing across looking-back conditions, with items serving as the unit of analysis (Table 6). The contribution of item differences to cued-recall performance was examined by including performance on single items in the model. Doing so was legitimate because items were rotated across conditions, such that the items representing the secondpresented instance of a category in the one-back and $n$-back conditions were the same as those representing the singleitem conditions, collapsed across participants. Performance on single items was entered as the first step of the model, and the contribution of recursive remindings was examined by entering the probability of producing P1 prior to the recalled instance, collapsed across spacing conditions, as

Table 6 Experiment 2: Proportions of variance in category-cued recall of second-presented instances of pairs explained by item differences, general memory ability, and looking back

\begin{tabular}{lll}
\hline & \multicolumn{2}{l}{ Unit of Analysis } \\
\cline { 2 - 3 } & Items & Participants \\
\hline Step 1 & & \\
$\quad$ Item differences/general memory & .04 & $.18^{*}$ \\
Step 2 & & $.26^{*}$ \\
Looking back & $.05^{*}$ & $.20^{*}$ \\
\hline
\end{tabular}

Values displayed above are $\Delta R^{2}$ on each step of the model computed at the item level collapsed across participants (left) and at the participant level collapsed across items (right). Item differences refers to item differences in single-item recall performance, general memory refers to individual differences in single-item recall performance, and looking back refers to differences in detection of shared category memberships for pairs. ${ }^{*} p<.05$. the second step. These predictor variables were entered in the specified order to estimate the extent to which recursive remindings predicted variance in the correct recall of $\mathrm{P} 2$ when controlling for item differences. Analyses were collapsed across looking-back and spacing conditions because neither variable produced any significant interactions. The results revealed that variance in cued-recall performance was weakly predicted by item differences $\left(\Delta R^{2}=.04, p=\right.$ $.09)$, but the explained variance was only marginally significant. When controlling for item differences, recursive remindings still explained unique variance in cued-recall performance $\left(\Delta R^{2}=.05\right)$, showing a small difference among items in their ability to support remindings. The small amount of variance explained by remindings is likely a result of the relationship between items being tightly controlled, and task control of remindings further reduced variability in remindings. Regardless, these results provide strong evidence that item-selection effects contributed extremely little to correct cued recall of P2.

Wahlheim and Jacoby (2013) had also used a hierarchical multiple regression analysis to examine the contribution of individual differences in the recollection of remindings to cued-recall performance, beyond individual differences in the general memory ability of participants. They had found cued-recall performance to increase with the probability of an individual recollecting remindings, as measured by the remindings-report procedure. We used a similar hierarchical multiple regression analysis to examine the effects of individual differences in the present experiment.

The regression model was the same that had been used to explore the contribution of item differences, except participants rather than items served as the unit of analysis (Table 6). We measured differences in general memory ability by examining differences in the correct recall of single items. Performance on single items was entered as the first step, and the probability of recursive remindings, as indicated by the remindings-report procedure, was entered as the second step. The predictor variables were entered in this order to estimate the extent to which individual differences in recursive remindings predicted variance in the correct recall of $\mathrm{P} 2$, beyond individual differences in general memory ability. The data were again collapsed across looking-back and spacing conditions because neither variable produced significant interactions. The results revealed that general memory performance accounted for a significant change in explained variance at the first step $\left(\Delta R^{2}=\right.$ .18 ), and that remindings accounted for a significant change at the second step when controlling for general memory differences $\left(\Delta R^{2}=.26\right)$. These results converge with the findings of Wahlheim and Jacoby (2013) in providing evidence for substantial individual differences in reliance on recursive remindings as a basis for cued-recall performance under conditions that require memory for temporal order. 
We found the contribution of individual differences in recursive remindings to cued-recall performance to be impressively large, although it was smaller than had sometimes been found by Wahlheim and Jacoby (2013). They found that the contribution of individual differences in reminding was smaller when the instructions were such as to direct remindings during List 2 study (Exp. 1), as compared to a condition in which remindings were not directed by the instructions. The $\Delta R^{2}$ accounted for by individual differences in recollection of remindings was .16 when remindings were directed during List 2, and .41 when they were not directed. In the present experiment, the materials employed, along with the $n$-back instructions, ensured that participants were largely successful at detecting the relationship between instances of a category.

The finding of individual differences in the contribution of recursive remindings to subsequent cued recall in the present experiments when encoding processes were so tightly controlled suggests that those individual differences arose from differences in reliance on recollection of recursive remindings at the time of test. Although recursive remindings were represented in memory, participants likely differed in their reliance on recollection of remindings either because they were less able to recollect those remindings or, instead, because the test strategy that they employed did not lead them to attempt such recollection.

\section{General discussion}

The results of our experiments show that recursive reminding can be brought under task control. Both recency judgments (Exp. 1) and cued recall of the most recently presented instance of a category (Exp. 2) were less accurate when remindings were discouraged by means of one-back instructions, rather than being encouraged by $n$-back instructions. The memory representation of recursive reminding embeds memory for the first-presented instance of a category $(\mathrm{P} 1)$ in the memory for the second-presented instance (P2), and by doing so preserves the order of their presentation and creates dependence between their earlier and later recall. Recollection that presentation of $\mathrm{P} 2$ produced a reminding of $\mathrm{P} 1$ serves to preserve their temporal order, which is important for both recency judgments and category-cued recall of the most recently presented instance of a category. When attempting to recall P2, participants in the $n$-back condition produced fewer intrusions of $\mathrm{P} 1$ than did those in the one-back condition when spacing of category instances was nonzero, as would be expected given the effect of looking-back instructions on recency judgments.

The remindings-report procedure measures recollection of recursive reminding as the probability of $\mathrm{P} 1$ being reported as coming to mind prior to or simultaneously with the item reported as being P2. Evidence that recursive remindings produced dependence in recall was provided by the finding that P1 was more likely in the $n$-back than in the one-back condition to be reported as coming to mind prior to the response produced as being P2 when the spacing of the instances was nonzero. In agreement with results reported by Wahlheim and Jacoby (2013), proactive facilitation was found when recursive reminding was recollected, whereas proactive interference was found when recursive reminding was not recollected. The results from a hierarchical regression analysis, along with other details of the results, serve to support the validity of the remindings-report procedure as a measure of the recollection of remindings.

The convergence of results gained by means of varying looking-back instructions and those gained by reliance on the remindings-report procedure supports our conclusions regarding the importance of recursive reminding. The remindings-report procedure relies on the use of conditional probabilities, and so it might be argued that its results are contaminated by item-selection effects. However, convergence of the results with those gained by varying lookingback instructions removes that worry, because examining the effects of looking back does not rely on the use of conditional probabilities. Also, the regression analysis showed that item differences contributed little to cuedrecall performance. In turn, it might be argued that the results gained by looking-back instructions reflect differences in study time rather than differences in recursive reminding. However, any differences in study time would necessarily be differences in effective study time, since the presentation rate during study was held constant at $5 \mathrm{~s}$ per item for the two looking-back conditions. Judgments during the presentation of the study list were faster in the one-back than in the $n$-back condition, though, and that difference could be viewed as corresponding to a difference in effective study time. Against an account in terms of differences in effective study time, differences between the one- and $n$ back conditions in response times were by far largest for the first-presented instance of a category. There, the manipulation of looking back condition produced only a main effect of decision time whereas our interest is in the interaction between looking back conditions and spacing of category instances. Furthermore, results from the remindings-report procedure did not rely on differences between the lookingback conditions, but converged with results gained by varying the looking-back instructions.

There is reason to expect a close relationship between proactive interference of the sort observed in Experiment 2 and proactive interference as it has traditionally been investigated. Proactive interference has traditionally been investigated by presenting items in two lists and asking for recall of the items presented in the second list (e.g., Postman \& Underwood, 1973). Judging the list membership of a pair is 
related to judgments of recency, because presented lists necessarily differ in their recency of presentation. This being the case, list discrimination can be seen as requiring a recency judgment, as did our Experiment 1, with the major difference that it requires discrimination of recency at the level of lists, whereas our experiments required a within-list discrimination of recency.

We interpret our results as showing that recollection of recursive reminding eliminates response competition by preserving the order in which potentially competing responses appeared. In contrast, list differentiation has traditionally been emphasized as serving to reduce response competition. Underwood $(1945,1949)$ used the notion of list differentiation to explain results that failed to show a high correlation between overt intrusions and retroactive interference. To explain the lack of correlation, Underwood suggested that participants employed a postretrieval editing process to recognize intrusions as coming from the wrong list, and thereby inhibit their emission. Winograd (1968) examined participants' ability to identify the list membership of previously studied items and discussed the importance of list differentiation as a means of avoiding interference effects.

When responses are changed between lists, both responses coming to mind at the time of test has been treated as being indicative of response competition, and the importance of list differentiation has been highlighted. In this vein, the modified-modified free recall test (MMFR) was designed to eliminate response competition by asking participants to recall responses from both lists and then to identify the list from which the responses originated (e.g., Barnes \& Underwood, 1959). In an A-B, A-B' paradigm for which responses in the two lists were strongly associated (e.g., afraid, scared), Barnes and Underwood found that recall was facilitated and that participants' ability to identify the list origins of responses was extremely high. The high list-identification performance is somewhat puzzling, since it was interpreted as resulting from list differentiation, and a strong association between responses would be expected to make such differentiation difficult to accomplish (cf. Young, 1955).

The rationale underlying the MMFR procedure is that interference effects result from response competition that is revealed by responses from both lists coming to mind at the time of test. The implication is that cued-recall performance should be poorer when both responses come to mind. In contrast, the results from our Experiment 2 showed that recall was higher when P1 was reported as coming to mind prior to the item given as being $\mathrm{P} 2$. For that case, proactive facilitation was found, rather than the proactive interference that response competition would be expected to produce. Response competition that was responsible for proactive interference relative to performance on control items was found only when no other response was reported as coming to mind ("No Other Reported" column in Table 5). It might be tempting to interpret these results as showing high temporal discrimination that is akin to high list differentiation. However, if one were to do so, the means of accomplishing list discrimination would have to be specified.

Although list differentiation can reduce response competition that serves as a source of proactive interference, the same can be accomplished by recursive reminding. The two means of avoiding the effects of response competition differ in important ways. Editing potential responses as a means of avoiding the interference effects produced by response competition relies on retrieval of temporal information or other details of the context in which an event occurred after possible responses have come to mind (e.g., Postman \& Underwood, 1973). According to that view, list membership is preserved by a list tag or context tag (see, e.g., J. R. Anderson \& Bower, 1974) that is associated with, but represented independently from, the representation of the content. In contrast, our results from the remindings-report procedure show that recursive remindings produce a dependence between the memory representations of events, and so influence what later comes to mind, as well as preserving temporal information. Recursive reminding produces a higher-order representation by embedding memory for one event in that of another event, rather than creating a simple association of an event with list or context tags.

Context tags in the form of a direct association between items and the list context in which they occurred could serve as a basis for temporal judgments. However, to explain the effects of looking back on subsequent cued-recall performance, one would have to argue that the retrieval of P1 produced by the presentation of $\mathrm{P} 2$ served to enhance subsequent temporal judgments by locking in the original context associated with P1. As we suggested earlier, one could as well argue that retrieval of $\mathrm{P} 1$ during the presentation of P2 would increase the difficulty of subsequent temporal judgments, by increasing the similarity of the context tags associated with the two items. Furthermore, it would have to be argued that people can only make judgments regarding temporal order by comparing context tags at the time of test, as they are unable to base temporal judgments on a higherorder relationship (a recursive reminding) that preserves temporal information. We believe that it is untenable to argue that people are unable to remember relationships, but instead are only capable of learning simple associations (for discussion relevant to this issue, see Asch, 1969).

According to a recursive-reminding account, temporal information is not represented separately as a simple association and accessed in an editing process after an item comes to mind. Rather, when an item is recollected, the recursive reminding that comes to mind with it, including temporalorder information, can be used for both recency judgments and cued recall of the most recently presented instances. Wahlheim and Jacoby (2013) described cases for which 
recursive reminding, rather than an influence on simple associations, might have been responsible for the effects observed in traditional investigations of proactive and retroactive interference (e.g., Barnes \& Underwood, 1959). Criss and Shiffrin (2005) showed that list discrimination can rely on higherorder relationships among events, rather than only relying on simple associations between items and the lists in which they occurred. As the researchers pointed out, several studies have attempted to distinguish a simple-associations assumption from a higher-order-relationships assumption, with results typically favoring the assumption of higher-order relationships (e.g., Hockley \& Cristi, 1996). Hintzman (2004) has described ways in which the results produced by recollection of remindings dictate changes in models of memory, including his own MINERVA model.

Our experiments extend arguments regarding the importance of remindings for recency judgments (Hintzman, 2011; Tulving, 1983, p. 42; Winograd \& Soloway, 1985) to account for proactive effects of memory. Our view of recursive reminding differs from that of Hintzman in that he (e.g., Hintzman, 2011) has described recursive reminding as generally being automatic, whereas we highlight the importance of task control and individual differences for the occurrence of recursive reminding. Our experiments provided little, if any, evidence of the occurrence of spontaneous reminding in the one-back condition. Participants in that condition seldom mistakenly responded "yes" during presentation of the second-presented instance of a category when spacing was nonzero. Also, in the one-back condition there was little evidence of spontaneous recursive reminding in subsequent recency judgments or cued recall when the spacing was nonzero. Although we agree that reminding can be relatively spontaneous, we doubt that reminding is ever fully divorced from the task in which one is engaged. In this vein, Ste-Marie and Jacoby (1993) provided evidence that spontaneity of recognition is relative to the task in which one is engaged (see also B. A. Anderson et al., 2011). What are the conditions that give rise to relatively spontaneous remindings? In part, this question can be answered by specifying situations in which remindings cannot be brought under task control. For example, it might be possible to find situations in which manipulating looking-back instructions does not fully eliminate spontaneous remindings in a condition that discourages their occurrence. Also, it is important to specify conditions that are necessary for recollection of remindings. The results of the present experiments suggest that even when reminding occurred during the presentation of the study list, there was a rather low probability of the remindings being recollected at the time of test. The measures of initial reminding and recollection of reminding in our experiments were very different, but the results do suggest frequent failures to recollect remindings (cf. Friedman, 2007).
Spontaneous remindings likely rely more heavily on the details of events, such as their physical similarity, than do directed remindings. Also, as was shown by the results of Experiment 2, there are large individual differences among people with regard to the contribution of recursive remindings to their performance (see also Jacoby, 1974; Wahlheim \& Jacoby, 2013). People who generally take a broad view and are credited with being creative may be subject to remindings that would not occur for those who generally take a more narrow view and, consequently, are blind to distant relationships among events. Such individual differences would have a variety of consequences. For example, those who take a broad view may be less prone to compartmentalization of knowledge (e.g., Potts, 1977) than are those who take a narrow view. On the other hand, a narrow view protects one from distraction and "scattered" thinking. Perhaps the detrimental effects of individual differences in openness to remindings could be greatly reduced by training. Can people be trained to more often engage in selfdirected processing of the sorts that encourage the occurrence and later recollection of recursive remindings? If so, what would be the costs and the benefits of such training? Perhaps it would be better to aim training at the balance between individual differences and task constraints. Are individual differences in openness to recursive remindings specific to particular domains or, instead, general across domains? These questions are among those that may serve as targets for future research.

The results of the present experiments provide evidence of the importance of recursive remindings produced by the detection of relationships between events. Earlier findings have shown that remindings resulting from detection of repetitions facilitate memory performance (e.g., Asch, 1969). Frequency judgments have also been shown to depend on recursive remindings (Hintzman, 2004). Recursive remindings resulting from the detection of change are important for later memory of paired associates presented in an A-B, A-D paradigm (Wahlheim \& Jacoby, 2013). Furthermore, remindings that accompany detection of change might play a role in memory for schema-inconsistent information about people (cf. Hastie \& Kumar, 1979). Remindings have also been said to play important roles in memory for more complex materials and concept learning (Benjamin \& Ross, 2010). Corballis (2011) argued that memory, thought, and language all rely on recursive processes.

A focus on the importance of recursive remindings gives rise to questions that are very different from those that come from treating memory as being fully reliant on simple associations, and has implications for applied purposes as well as for theory. An important difference between approaches is that the notion of remindings highlights the importance of an awareness of relationships, whereas questions of awareness have not been given a prominent role in simple- 
association accounts of the sort that have dominated theorizing about the retroactive and proactive effects of memory (for a discussion of this point, see Wahlheim \& Jacoby, 2013). The results of our experiments, along with those reported by others, provide evidence that the detection and later recollection of relationships among events are important for exploiting those relationships. In line with the quote at the top of this article, life can only be understood by looking back, but looking back can facilitate moving forward.

Author note This research was supported by a James S. McDonnell Foundation 21st Century Science Initiative in Bridging Brain, Mind, and Behavior Collaborative Award to the first author. We thank Emily Gardner and Rachel Teune for their assistance with manuscript preparation, Ashley Bartels and Rachel Teune for their assistance with data collection, and Mike Strube for his assistance with data analysis. We also thank Colleen Kelley for her comments on an earlier draft of this article.

\section{References}

Anderson, J. R., \& Bower, G. H. (1974). Interference in memory for multiple contexts. Memory \& Cognition, 2, 509-514.

Anderson, B. A., Jacoby, L. L., Thomas, R. C., \& Balota, D. A. (2011). The effects of age and divided attention on spontaneous recognition. Memory \& Cognition, 39, 725-735. doi:10.3758/s13421010-0046-z

Anderson, M. C., \& Neely, J. H. (1996). Interference and inhibition in memory retrieval. In E. L. Bjork \& R. A. Bjork (Eds.), Handbook of perception and cognition: Memory (2nd ed., pp. 237-313). San Diego, CA: Academic Press.

Appleton-Knapp, S. L., Bjork, R. A., \& Wickens, T. D. (2005). Examining the spacing effect in advertising: Encoding variability, retrieval processes, and their interaction. Journal of Consumer Research, 32, 266-276.

Asch, S. E. (1969). A reformulation of the problem of associations. American Psychologist, 24, 92-102.

Barnes, J. M., \& Underwood, B. J. (1959). "Fate" of first-list associations in transfer theory. Journal of Experimental Psychology, 58, 97-105. doi:10.1037/h0047507

Bellezza, F. S., Winkler, H. B., \& Andrasik, F. (1975). Encoding processes and the spacing effect. Memory \& Cognition, 3, 451457. doi:10.3758/BF03212940

Benjamin, A. S., \& Ross, B. H. (2010). The causes and consequences of reminding. In A. S. Benjamin (Ed.), Successful remembering and successful forgetting: A festschrift in honor of Robert A. Bjork (pp. 71-87). New York, NY: Psychology Press.

Benjamin, A. S., \& Tullis, J. G. (2010). What makes distributed practice effective? Cognitive Psychology, 61, 228-247.

Berntsen, D., Staugaard, S. R., \& Sørensen, L. M. T. (2013). Why am I remembering this now? Predicting the occurrence of involuntary (spontaneous) episodic memories. Journal of Experimental Psychology: General. doi:10.1037/a0029128

Braun, K., \& Rubin, D. C. (1998). The spacing effect depends on encoding deficit, retrieval and time in working memory: Evidence from once-presented words. Memory, 6, 37-65.

Corballis, M. C. (2011). The recursive mind: The origin of human language, thought, and civilization. Princeton, NJ: Princeton University Press.
Criss, A. H., \& Shiffrin, R. M. (2005). List discrimination in associative recognition and implications for representation. Journal of Experimental Psychology: Learning, Memory, and Cognition, 31, 1199-1212. doi:10.1037/0278-7393.31.6.1199

Friedman, W. J. (2007). The role of reminding in long-term memory for temporal order. Memory \& Cognition, 35, 66-72.

Hastie, R., \& Kumar, P. A. (1979). Person memory: Personality traits as organizing principles in memory for behaviors. Journal of Personality and Social Psychology, 37, 25-38.

Hintzman, D. L. (2004). Judgment of frequency versus recognition confidence: Repetition and recursive reminding. Memory \& Cognition, 32, 336-350. doi:10.3758/BF03196863

Hintzman, D. L. (2010). How does repetition affect memory? Evidence from judgments of recency. Memory \& Cognition, 38, 102-115. doi:10.3758/MC.38.1.102

Hintzman, D. L. (2011). Research strategy in the study of memory: Fads, fallacies, and the search for the "coordinates of truth. Perspectives on Psychological Science, 6, 253-271. doi: $10.1177 / 1745691611406924$

Hockley, W. E., \& Cristi, C. (1996). Tests of the separate retrieval of item and associative information using a frequency-judgment task. Memory \& Cognition, 24, 796-811.

Jacoby, L. L. (1974). The role of mental contiguity in memory: Registration and retrieval effects. Journal of Verbal Learning and Verbal Behavior, 13, 483-496. doi:10.1016/S0022-5371(74)80001-0

Kierkegaard, S. (1996). Papers and journals: A selection (A. Hannay, Trans.). London, UK: Penguin. (Original work published 1869).

Postman, L., \& Underwood, B. J. (1973). Critical issues in interference theory. Memory \& Cognition, 1, 19-40.

Potts, G. R. (1977). Integrating new and old information. Journal of Verbal Leaning and Verbal Behavior, 16, 305-320.

Ste-Marie, D. M., \& Jacoby, L. L. (1993). Spontaneous versus directed recognition: The relativity of automaticity. Journal of Experimental Psychology: Learning, Memory, and Cognition, 19, 777-788. doi:10.1037/0278-7393.19.4.777

Tulving, E. (1983). Elements of episodic memory. New York, NY: Oxford University Press.

Tzeng, O. J. L., \& Cotton, B. (1980). A study-phase retrieval model of temporal coding. Journal of Experimental Psychology: Human Learning and Memory, 6, 705-716.

Underwood, B. J. (1945). The effect of successive interpolations on retroactive and proactive inhibition. Psychological Monographs, $59,1-33$.

Underwood, B. J. (1949). Proactive inhibition as a function of time and degree of prior learning. Journal of Experimental Psychology, 39, 24-34.

Van Overschelde, J. P., Rawson, K. A., \& Dunlosky, J. (2004). Category norms: An updated and expanded version of the Battig and Montague (1969) norms. Journal of Memory and Language, 50, 289-335. doi:10.1016/j.jml.2003.10.003

Wahlheim, C. N., \& Jacoby, L. L. (2013). Remembering change: The critical role of recursive remindings in proactive effects of memory. Memory \& Cognition, 41, 1-15. doi:10.3758/s13421-012-0246-9

Winograd, E. (1968). List differentiation as a function of frequency and retention interval. Journal of Experimental Psychology, 76(2, Pt. 2), 1-18. doi:10.1037/h0025380

Winograd, E., \& Soloway, R. M. (1985). Reminding as a basis for temporal judgments. Journal of Experimental Psychology: Learning, Memory, and Cognition, 11, 262-271.

Yonelinas, A. P., \& Jacoby, L. L. (2012). The process-dissociation approach two decades later: Convergence, boundary conditions, and new directions. Memory \& Cognition, 40, 663-680. doi:10.3758/s13421-012-0205-5

Young, R. K. (1955). Retroactive and proactive effects under varying condition of response similarity. Journal of Experimental Psychology, 50, 113-119. 